Check for updates

The BMJ

Cite this as: $B M J 2020 ; 370: m 3682$ http://dx.doi.org/10.1136/bmj.m3682 Published: 18 September 2020

\section{Covid-19: Point of care test reports $94 \%$ sensitivity and $100 \%$ specificity compared with laboratory test}

\author{
Elisabeth Mahase
}

A point of care covid-19 test-which the UK government plans to roll out across the country-can provide results in 90 minutes with $94 \%$ sensitivity and $100 \%$ specificity when compared with standard polymerase chain reaction (PCR) testing, according to a study from the manufacturers. ${ }^{1}$

The study, published in Lancet Microbe, tested the CovidNudge real time PCR platform between 2 April and 18 May 2020 at three London hospitals, with 449 same day samples collected. Two swabs were taken per person, with the samples then being tested in parallel using the point of care test and the standard PCR test.

Researchers have said that the test can quickly tell whether patients are infected. Currently, laboratory based PCR testing takes around four to six hours, and when transportation of clinical samples is factored in the turnaround time often exceeds 24 hours, which can result in "delays in diagnoses and inappropriate infection control precautions," the authors said.

The CovidNudge test does not require laboratory processing. Lead author of the study, Graham Cooke, stressed, however, that this test is not likely to be used for mass testing at events attended by thousands of people because the machine can only process one test at a time, with a maximum of 15 tests per machine each day.

The UK government has placed an order for $5.8 \mathrm{~m}$ of the testing kits, which it intends to roll out across the nation. ${ }^{2}$ The test was mentioned in the leaked Moonshot documents, revealed by The BMJ, as part of the government's plan to test the whole population every week from early $2021 .^{3}$

The CovidNudge test involves a nasopharyngeal swab, which is inserted directly into a cartridge containing all reagents and components required for RT-PCR reactions.

The study included complete clinical data paired with laboratory tests for 386 samples, of which, 67 tested positive on the CovidNudge point of care platform and 71 with standard laboratory RT-PCR. The overall sensitivity of the point of care test compared with laboratory based testing was 94\% (95\% confidence interval 86 to 98 ) with an overall specificity of $100 \%$ (99 to 100), reported researchers.

One key feature of the test is the inclusion of human ribonuclease $\mathrm{P}$ control in the testing cartridge, which helps ensure that the sample is good enough to be tested. Speaking at the Science Media Centre briefing on the study, Cooke, professor of infectious diseases at Imperial College London, said, "We hear a lot about false negatives, a lot of these are actually because the test has not been taken well enough. This has a positive control on it so you can tell if someone has taken the test well enough."

He added, "We have run over 10 ooo tests now in clinical practice and people are comfortable with using it. We think this is a useful part of the whole puzzle for diagnostics. This is well sited for clinical settings where you are trying to make a rapid decision for a patient."

Lawrence Young, professor of molecular oncology at the University of Warwick, said the CovidNudge test could have an important role where real time decision making was necessary, such as screening patients for admission to hospital or for surgery. But he said that the test "needs further evaluation in comparison with standard laboratory tests to determine whether it can be reliably used to measure the level of virus infection in an infected person."

The researchers have recommended that local assessments are carried out to compare performance against local standards of care when the device is first deployed in a new setting.

The study was funded by the National Institute of Health Research and DnaNudge, the company behind the technology.

Gibani MM, Toumazou C, Sohbati M, et al. Assessing a novel, lab-free, point-of-care test for SARS-CoV-2 (CovidNudge): a diagnostic accuracy study. Lancet Microbe. 17 September 2020. www.thelancet.com/journals/lanmic/article/PIIS2666-5247(20)30121-X/fulltext.

Mahase E. Covid-19: UK hospitals to get rapid tests from September. BMJ 2020;370:m3087. doi: 10.1136/bmj.m3087 pmid: 32753401

3 Mahase E. Operation Moonshot: Testing plan relies on technology that does not exist. BMJ 2020;370:m3585.

doi: 10.1136/bmj.m3585 pmid: 32938606 